\title{
A National Survey of Undergraduate Clinical Education in Internal Medicine
}

\author{
Amber T. Pincavage, $M D^{7}$, Mark J. Fagan, $M D^{2}$, Nora Y. Osman, $M D^{3}$, \\ Debra S. Leizman, $M D^{4}$, Deborah DeWaay, $M D^{5}$, \\ Camilla Curren, $M D^{6}$, Nadia Ismail, $M D^{7}$, Karen Szauter, $M D^{8}$, \\ Michael Kisielewski, $M A^{9}$, and Amy W. Shaheen, $M^{10}$
}

\begin{abstract}
'Department of Medicine, University of Chicago Pritzker School of Medicine, Chicago, IL, USA; ${ }^{2}$ Department of Medicine, Alpert Medical School of Brown University, Providence, RI, USA; ${ }^{3}$ Department of Medicine, Brigham and Women's Hospital, Harvard Medical School, Boston, MA, USA; ${ }^{4}$ Department of Medicine, University Hospital Cleveland Medical Center, Case Western Reserve University School of Medicine, Cleveland, OH, USA; ${ }^{5}$ Department of Internal Medicine, Morsani College of Medicine, University of South Florida, Tampa, FL, USA; ${ }^{6}$ Department of Internal Medicine, The Ohio State University College of Medicine, Columbus, OH, USA; ${ }^{7}$ Department of Medicine, Baylor College of Medicine, Houston, TX, USA; ${ }^{8}$ Department of Internal Medicine, University of Texas Medical Branch, Galveston, TX, USA; ${ }^{9}$ Alliance for Academic Internal Medicine, Alexandria, VA, USA; ${ }^{10}$ Department of Internal Medicine, The University of North Carolina, Chapel Hill, NC, USA.
\end{abstract}

BACKGROUND: In the present milieu of rapid innovation in undergraduate medical education at US medical schools, the current structure and composition of clinical education in Internal Medicine (IM) is not clear.

OBJECTIVE: To describe the current composition of undergraduate clinical education structure in IM.

DESIGN: National annual Clerkship Directors in Internal Medicine (CDIM) cross-sectional survey.

PARTICIPANTS: One hundred twenty-nine clerkship directors at all Liaison Committee on Medical Education accredited US medical schools with CDIM membership as of September 1, 2017.

MAIN MEASURES: IM core clerkship and post-core clerkship structure descriptions, including duration, educational models, inpatient experiences, ambulatory experiences, and requirements.

KEY RESULTS: The survey response rate was 83\% (107/ 129). The majority of schools utilized one core IM clerkship model (67\%) and continued to use a traditional block model for a majority of their students (84\%). Overall $26 \%$ employed a Longitudinal Integrated Clerkship model and $14 \%$ employed a shared block model for some students. The mean inpatient duration was $7.0 \pm 1.7$ weeks (range 3-11 weeks) and 94\% of clerkships stipulated that students spend some inpatient time on general medicine. IMspecific ambulatory experiences were not required for students in $65 \%$ of IM core clerkship models. Overall $75 \%$ of schools did not require an advanced IM clinical experience after the core clerkship; however, $66 \%$ of schools reported a high percentage of students (>40\%) electing to take an IM sub-internship. About half of schools (48\%) did not require overnight call or night float during the clinical IM sub-internship.

CONCLUSIONS: Although there are diverse core IM clerkship models, the majority of IM core clerkships are still

Prior Presentations Initial data from this study was presented at the National 2018 CDIM meeting March 2018 in San Antonio, TX.

Electronic supplementary material The online version of this article (https://doi.org/10.1007/s11606-019-04892-0) contains supplementary material, which is available to authorized users.

Published online April 16, 2019 traditional block models. The mean inpatient duration is 7 weeks and $65 \%$ of IM core clerkship models did not require IM-specific ambulatory education.

KEY WORDS: undergraduate medical education; clerkship; internal medicine clerkship.

J Gen Intern Med 34(5):699-704

DOI: $10.1007 / \mathrm{s} 11606-019-04892-0$

(c) Society of General Internal Medicine 2019

\section{INTRODUCTION}

Medical education is changing rapidly with increasing class sizes and numbers of medical schools in order to meet the physician shortage, increasing accreditation demands, and a call for education reform. ${ }^{1-5}$ Clinical training is beginning earlier and clinical experiences are adjusting to accommodate larger class sizes, student demand, increased curricular content, and accreditation standards. ${ }^{1,5,6}$ As a result, it is increasingly difficult to determine how educational models have evolved given these challenges. The curriculum inventory initiative from the Association of American Medical Colleges (AAMC) has attempted to provide details about clinical courses; however, the data are not granular enough to provide details on course structure and schools have varying definitions of the courses. ${ }^{7}$

Additional pressures on traditional models of education include a shift toward competency-based education and an increased emphasis by the AAMC and Liaison Committee on Medical Education (LCME) on ambulatory training. As patient care has shifted more to outpatient settings, training in outpatient settings has increasingly been prioritized. ${ }^{4,8-12}$ The LCME now stipulates medical schools meet ambulatory learning requirements to ensure trainees are well prepared for outpatient practice. ${ }^{4}$ Likewise, the AAMC has outlined competencies that are specific to ambulatory education. ${ }^{8}$ In response to competencybased education and increased ambulatory education, Hirsh et al. have advocated for clinical models that enhance continuity with 
faculty preceptors and patients. ${ }^{13}$ Particularly, longitudinal integrated clerkship (LIC) models, models with a combination of several core clinical clerkships across disciplines integrated into one longitudinal experience, have been piloted and advocated by the Consortium of LICs to improve educational continuity, ambulatory education, and competency-based assessment. ${ }^{14-16}$ Clerkship shared block models, with a combination of medical disciplines integrated into one core rotation block, have also been described. ${ }^{17}$ Shared block models have been particularly common for primary care clerkships which may combine Family Medicine, Internal Medicine (IM), Psychiatry, and other ambulatory-based specialty training into one clerkship block. These have been created to accommodate increased ambulatory learning requirements and increased numbers of students in addition to other factors. ${ }^{17}$ Additionally, with the advent of the educational goal of entrustment to meet the AAMC's Core Entrustable Professional Activities for Entering Residency (CEPAERs), more attention is being paid to post-clerkship fourth-year clinical training. ${ }^{8,18-21}$

These national-level medical education initiatives and reforms have impacted and spawned further changes in clinical undergraduate medical education (UME) IM training both during and after the core clerkship. ${ }^{17}, 22$ However, the last national survey by the Clerkship Directors in Internal Medicine (CDIM) evaluating core clerkship IM training occurred in 2010 and in 2008 to evaluate IM sub-internship training. ${ }^{12,23}$ These surveys occurred prior to the publication of the CEPAERs, increased focus on ambulatory education, and widespread promotion of LIC's; thus, the degree to which clinical training in IM has changed in response to recent curricular reform efforts is not clear.

In order to assess the current composition of IM clinical education structure in UME, the CDIM Scholarship and Survey Committee surveyed members about the structure of clinical IM education in 2017. In this paper, we summarize the results of the 2017 CDIM survey on the current structure of IM clinical education. By comparing these results to previous CDIM survey data, we describe temporal changes and consider implications of these results.

\section{METHODS}

On September 14, 2017, CDIM launched its annual, voluntary, and confidential survey of clerkship directors (CD) at all LCME-accredited US medical schools with current CDIM membership. Altogether, 131 CDIM members designated as "clerkship director" received a personal e-mail invitation to complete the web-based survey. Only one individual per member school received the invitation. About 90\% (131/ 147) of LCME-accredited schools were represented in CDIM during the survey period.

The survey questions were developed by a nine-person subcommittee of the CDIM Survey and Scholarship Committee during 2017. The questions were written in reference to past CDIM Annual Survey questions using best practices of survey design. The questions were reviewed and modified by the committee over the course of 2 months after multiple pilot tests. The committee further revised the survey after the elected CDIM Council reviewed it; the Council and the survey committee were composed of CDIM members who were the survey target population and expert stakeholders. The final survey consisted of 39 questions on clerkship structure of different types, including multiple-choice, numeric-only, and open-text response options, and included logical skip and display patterns (supplement).

The survey was administered via Qualtrics survey software using Secure Socket Layer (SSL) encryption and included five e-mail reminders to non-respondents. During fielding, the population size was adjusted to 129 possible respondents, due to invalid contact information. The survey closed on December 5, 2017. The survey protocol was granted full institutional review board exempt status from the University of North Carolina Office of Human Research Ethics.

\section{Data Analysis}

Data analysis was performed in Stata $14.2,{ }^{24}$ and included descriptive statistics and statistical significance tests for groupbased differences, using the Pearson chi-square statistic or Fisher's exact test, as appropriate. Following data collection, a variable to denote respondents' and non-respondents' medical school as "public" or "private" was merged into the dataset, using publicly available data and visits to medical school websites. ${ }^{25}$ Using CDIM membership files, data on respondents' and non-respondents' gender and US Census Bureau geographic region of their school also were merged into the dataset. ${ }^{26}$ All respondent survey contact information was handled by MK. Upon survey closure, all survey data were downloaded to a local network drive accessible only to MK, and then deleted from the web and de-identified prior to analysis. No other coauthors had access to the survey dataset prior to de-identification. Due to the high survey response rate and lack of statistically significant differences between survey respondents and non-respondents, the data were not weighted to adjust for nonresponse. Due to item non-response, survey conditional logic, or the ability to provide multiple responses to certain questions, some denominators vary and do not sum to 107 .

\section{RESULTS}

The overall response rate was $83 \%$ (107/129). There were no statistically significant differences between respondents and non-respondents based on the three variables merged into the dataset after fielding: medical school type (public/private), US Census Bureau region, and gender of respondent (Table 1).

\section{Overall Structure}

At $92.5 \%$ of responding institutions (99/107), students commence clinical clerkships at the same stage of medical school 
Table 1 Respondent and Non-respondent Characteristics: 2017 CDIM Annual Survey of Clerkship Directors

\begin{tabular}{|c|c|c|c|}
\hline & \multirow{2}{*}{$\begin{array}{l}\text { Respondents } \\
N=107 \\
n(\%)\end{array}$} & \multirow{2}{*}{$\begin{array}{l}\text { Non-respondents } \\
N=24 \\
n(\%)\end{array}$} & \multirow[t]{2}{*}{$p$ value } \\
\hline & & & \\
\hline \multicolumn{4}{|l|}{ Gender } \\
\hline Female gender & $52(49.1) * *$ & $10(41.7)$ & 0.51 \\
\hline \multicolumn{4}{|l|}{ Institution type } \\
\hline Public & $65(60.8)$ & $18(75.0)$ & 0.19 \\
\hline Private & $42(39.3)$ & $6(25.0)$ & 0.19 \\
\hline \multicolumn{4}{|l|}{ Institution region } \\
\hline Northeast & $27(25.2)$ & $6(25.0)$ & 0.98 \\
\hline Midwest & $27(25.2)$ & $5(20.8)$ & 0.80 \\
\hline Southern & $38(35.5)$ & $10(41.7)$ & 0.57 \\
\hline Western & $15(14.0)$ & $2(8.3)$ & 0.74 \\
\hline \multicolumn{4}{|l|}{ Class size } \\
\hline$<50$ students & $2(1.9)$ & - & - \\
\hline 51-80 students & $10(9.3)$ & - & - \\
\hline $81-120$ students & $21(19.6)$ & - & - \\
\hline $121-200$ students & $55(51.4)$ & - & - \\
\hline$>200$ students & $19(17.8)$ & - & - \\
\hline \multicolumn{4}{|c|}{ Minimum pre-clinical months } \\
\hline $1-11$ & $1(0.9)$ & - & - \\
\hline 12 & $2(1.9)$ & - & - \\
\hline $13-18$ & $33(30.8)$ & - & - \\
\hline 19-24 & 69 (64.5) & - & - \\
\hline$>24$ & $1(0.9)$ & - & - \\
\hline
\end{tabular}

*Pearson chi-square test used for categorical variables; Fisher's exact test used when expected cell counts are less than five

**One missing data point: $n=106$

(Table 1). No schools identified varying student clinical clerkship start time based on milestone completion and student choice was the most common cited reason for variation.

Over the past 5 years, $57.9 \%(62 / 107)$ of respondents reported that there was no change in the start time of the clinical training, 37.3\% (40/107) of respondents started clinical training earlier, and 1.9\% (2/107) started clinical training later.

Most medical schools organized the IM clerkship using a Traditional Model (an IM block) for at least some of their students followed by the LIC Model and then the least number of schools used the shared block model (Table 2). Overall 67\% of schools had only one core IM clerkship model (72/107). Of

Table 2 Internal Medicine Core Clerkship Models: 2017 CDIM Annual Survey of Clerkship Directors

\begin{tabular}{ll}
\hline \hline & $\begin{array}{l}\text { Number of institutions (\%) } \\
\text { N=107 }\end{array}$ \\
\hline $\begin{array}{l}\text { Traditional block } \\
\text { Traditional block only }\end{array}$ & $59(55.1)$ \\
Any traditional block & $90(84.1)$ \\
LIC model & $1(0.9)$ \\
LIC only & $28(26.2)$ \\
Any LIC & $10(9.3)$ \\
Shared block & $15(14.0)$ \\
Shared block only & $22(20.6)$ \\
Any shared block & $2(1.9)$ \\
Mixed models & $3(2.8)$ \\
Traditional block and LIC & $2(1.9)$ \\
Traditional block and shared block & $2(1.9)$ \\
Traditional block and LIC and other & $1(0.9)$ \\
LIC and shared block & \\
Traditional block and other & Shared block and other
\end{tabular}

those schools with more than one core IM clerkship model (32\%, 35/107), 43\% (15/35) assigned students in different structural models to rotate at the same clinical sites and $46 \%$ $(16 / 35)$ did not.

\section{Inpatient Structure}

All respondents reported that students spent time on the inpatient service as part of the IM core clerkship. The amount of time spent on inpatient medicine during the clerkship over the past 5 years remained constant in $77 \%$ of institutions $(82 / 107)$, decreased in 20\% (21/107), and increased in 2\% (2/107). The mean inpatient time was $7.0 \pm 1.7$ weeks (range 3-11 weeks) with the most common duration of 7-8 weeks $(57 \%, 61 / 107)$ or 5-6 weeks $(23 \%, 25 / 107)$. Inpatient IM time was lowest in the LIC model (Table 3). Students spent some time on an inpatient general medicine service in $94 \%$ of clerkships (100/ 107). Students were less inclined to spend time on a subspecialty service with $53 \%$ of clerkships (57/107) offering no subspecialty experience. When subspecialty experiences were offered, students spent time on diverse subspecialty services with cardiology, oncology, infectious disease, and geriatrics designated as the most popular assignments.

Overall 22\% (24/107) of inpatient IM clerkships added new sites in the past 5 years, $13 \%(14 / 107)$ consolidated to fewer sites, and 63\% (67/107) reported no changes in the numbers of teaching sites. The number of sites was lowest for LIC models (Table 3). The quantity of sites changed for various reasons but the top-four reasons included gained resources, resources lost, competing professional students, and a change in class size.

\section{The Structure of Ambulatory Learning}

Respondents reported that IM-specific ambulatory experiences were not required for students in $65.4 \%$ (70/107) of IM core clerkship models. The curricular model for an individual student's core clerkship had the greatest impact on whether a student spent time learning IM in an ambulatory setting. If the student's core IM clerkship was part of a LIC or shared block, fewer respondents reported including required learning in an IM-specific ambulatory setting (Table 3). While the traditional block model respondents more often reported their students were required to spend time in an IM-specific ambulatory setting, the actual number of half days spent in ambulatory medicine was greatest in the LIC as compared to the traditional block model (Table 3).

\section{Post-core Clerkship}

After the core clerkship, $74.8 \%$ of schools (80/107) did not require an advanced IM clinical experience. Of those requiring some form of IM experience $(23 \%, 25 / 107)$, the required clinical experiences were sub-internships (36\%, 9/25), ambulatory rotations $(32 \%, 8 / 25)$, inpatient IM rotations $(24 \%$, $6 / 25)$, or ICU experiences $(20 \%, 5 / 25)$. For $68 \%$ of respondents (17/25), the amount of time required for mandatory post- 
Table 3 IM Core Clerkship Model Comparisons: 2017 CDIM Annual Survey of Clerkship Directors

\begin{tabular}{lll}
\hline \hline & $\begin{array}{l}\text { Traditional block } \\
(\boldsymbol{N}=\mathbf{8 9}) \text { mean } \pm \text { SD }\end{array}$ & $\begin{array}{l}\text { LIC } \\
(\boldsymbol{N}=\mathbf{1 9}) \mathbf{m e a n} \pm \mathbf{S D} \\
(\boldsymbol{N}=\mathbf{1 4}) \mathbf{m e a n} \pm \mathbf{S D}\end{array}$ \\
\hline Percent students per class & $63 \pm 38.1(n=86)$ & $18 \pm 22.6$ \\
Length in weeks & $9.2 \pm 5.1(n=86)$ & $36.6 \pm 15.6$ \\
Weeks of inpatient & $7.0 \pm 1.6(n=88)$ & $4.7 \pm 2.3(n=17)$ \\
Sites & $4.2 \pm 3.6$ & $2.8 \pm 2.6$ \\
Maximum number of students per rotation & $32.1 \pm 25.1(n=86)$ & $18.8 \pm 17.2(n=18)$ \\
Ambulatory IM time required x/n $(\%)$ & $45 / 101(44)$ & $20 / 60(33)$ \\
Minimum half days in IM ambulatory & $15.0 \pm 10.1(n=41)$ & $31.3 \pm 27.7(n=17)$ \\
\hline
\end{tabular}

Because schools may have multiple models within the same school, the number of responses exceeded the number of respondents; multiple responses were allowed

clerkship IM experiences remained the same over the past 5 years, while it decreased for $20 \%(5 / 25)$ of schools.

Only $13 \%(14 / 107)$ of schools required a clinical subinternship in IM to graduate; however, $66 \%$ (61/92) of schools reported that over $40 \%$ of students elected to take one. Eightysix percent of schools (92/107) designed their curricula to allow students to begin their sub-internship at the beginning of their fourth year. Descriptions of IM sub-internships varied and included options such as night float, general medicine wards, ambulatory-based rotations, and ICU. Thirteen percent of institutions (14/107) required an ambulatory clerkship after core clerkships, although some of these were not in IM. Overnight call $(12 \%, 12 / 102)$ and night float $(14 \%, 14 / 102)$ were required in some institutions. For others, night call was optional $(14 \%, 14 / 102)$, and $48 \%(51 / 107)$ of institutions did not require it.

\section{DISCUSSION}

Our study demonstrates that the diversity of IM core clerkship models have increased although the majority are still traditional block models. Additionally, the majority of IM core clerkships are not requiring IM-specific ambulatory education. Although the majority of institutions reported no changes in the IM core clerkship inpatient duration or the duration of mandatory post-clerkship IM experiences in the past 5 years, data from older studies demonstrate changes have occurred. As we compare our results to previous data regarding clinical IM education, it suggests exposure to clinical IM education has decreased, including decreased IM inpatient time, decreased dedicated IM-specific ambulatory time, and decreased post-core clerkship IM experience and most of this decline occurred more than 5 years ago. Overall, several local and national priorities explain this evolution in IM clinical education. One likely factor is the effect of increasing class size on education and training capacity. The AAMC advocated for increasing class size to help address the predicted physician shortage $^{1-3}$ and concordantly, a little more than half of our respondents reported a class size of 121-200 students, with $17.8 \%$ reporting class sizes of more than 200 students. In comparison, the 2006 CDIM Annual Survey found an average class size of 140 students. ${ }^{17}$ In order to increase capacity-as exemplified by our data - schools have incorporated additional models and shared resources with other specialties.

Another major factor is the increased demand for ambulatory training capacity for medical students. In order to support increasing numbers of students and increased ambulatory education requirements, more ambulatory sites and preceptors are needed. Ambulatory preceptor recruitment and retention has been problematic for many IM educators and clerkships. ${ }^{27-29}$ Thus, in an effort to accommodate the increased capacity for ambulatory education for more students, the education has migrated from IM to interdisciplinary primary care, which includes non-IM specialties. So, although ambulatory training has increased overall, IM-specific ambulatory education has decreased. This is echoed by our results. In the 2010 CDIM Annual Survey, 72\% (57/79) of respondents reported that ambulatory education was a required part of the core IM clerkship, as opposed to the current $35 \%$ of IM core clerkship models requiring it. ${ }^{12}$

We also suspect that a major cause of the compression of IM exposure and specifically inpatient IM time has been an effort to accommodate increased ambulatory education in addition to a growing amount of diverse curricula and new topics into UME. $^{12,17,27}$ The numbers of requirements and topics to address in UME have increased without additional time expansion and have included innovative topics such as patient safety, high value care, and transitions of care, which have been emphasized in the CEPAER's. ${ }^{8,} 27$ In response to these competing demands, the IM inpatient time has been compressed even more than previously described. Compared to the 2010 CDIM survey data, only $6.5 \%$ of respondents in 2017 indicated greater than 8 weeks of inpatient time as opposed to $17 \%$ in $2010 .{ }^{12}$ Likewise, there was an increase in the number of clerkships with 5 to 6 weeks of inpatient time from $9 \%$ in 2010 to $23 \%$ in $2017 .^{12}$ As more has been added into UME, IM exposure has dwindled.

Our study also demonstrates a de-emphasis of the IM subinternship in UME. Our finding that $13 \%$ of schools require a sub-internship in IM represents a substantial decrease from 2000 , when a survey found that $26 \%$ of schools required an IM sub-internship. ${ }^{30}$ This decline has occurred despite the educational importance attributed to an IM sub-internship by 
IM CDs, ${ }^{18}$ multi-specialty residency program directors (PDs), ${ }^{31}$ and IM residents. ${ }^{32}$ Aiyer et al. stated that the IM sub-internship "...with its strong emphasis on experiencebased learning and increased patient responsibilities, is an important component of undergraduate education." 23 LyssLerman et al. conducted semi-structured interviews with 30 multi-specialty residency PDs and found "...a consensus across specialties that fourth year students should pursue sub-internships in their future fields, in IM, and in an IM subspecialty". ${ }^{31}$ Sixty-three percent of PDs specifically recommended an IM sub-internship. In a position paper, the CDIM-Association of Program Directors in IM (APDIM) Committee on Transitions to Internship Group describes how the goals of clinical IM education align with the AAMC CEPAERs for entering residency. ${ }^{8}, 18$ Despite this consensus from multiple perspectives regarding the pivotal role of IM clinical training and its congruence with the CEPAERs, it is surprising that exposure to clinical IM training has decreased.

Additionally, there has been a reduction in night call and night experiences during the IM sub-internship over time. A 2000 survey found that $77 \%$ of IM sub-internships included integration of sub-interns into call schedules, which would have been overnight call in the pre-duty hours era. ${ }^{30}$ The 2014 CDIM Annual Survey found that $40.3 \%$ of institutions had sub-interns take overnight call compared to the $12 \%$ of institutions currently requiring it. ${ }^{33}$ This likely reflects the Accreditation Council for Graduate Medical Education (ACGME) 2011 duty hour regulations restricting interns to $16 \mathrm{~h}$ of call. ${ }^{34,35}$ However, with the recent relaxation of intern duty hours by the ACGME, our data suggest that many trainees will experience their first night experience as an intern with higher stakes and less supervision. ${ }^{36}$ Advocacy for additional preparation for night call should be considered prior to and during internship.

We are concerned about these findings for several reasons. Less exposure to IM in UME may decrease interest and recruitment into the field. ${ }^{37}$ Particularly, the decreased exposure to ambulatory IM may decrease recruitment into primary care IM careers, which already struggle to match the needs of the population. Additionally, IM education has been uniquely poised to teach clinical reasoning and care of complex adult patients to medical students, competencies that are integral to multiple specialties and have been coveted by many specialties. $^{31,37}$ We are concerned that with declined exposure to IM, the training of our future physicians will suffer. We are also concerned that the reduction of night call and night experiences in IM sub-internships will diminish the preparedness of our students for IM residencies. In the near future, it will be critical to examine the effects of these changes on trainees and to advocate for adequate IM clinical education to meet the expectations we have for medical students to be ready for GME training, and to recruit an adequate workforce into IM. IM educators and departments need to be aware of these trends before opportunities to engage with learners and curricular space are further controlled by other departments.
We have several limitations in our study. Due to the high response rate and lack of statistically significant differences between respondents and non-respondents for the three variables merged into the survey dataset after fielding, the data were not weighted to adjust for survey non-response. However, it is possible that other individual member or medical school characteristics were not considered which might demonstrate differences. Additionally, the item response rate varied for certain questions, which could be due to survey fatigue or due to certain items being non-applicable to certain respondents. This variable response could have biased our results for questions with lower response. Our findings are based on self-report of CDs, which may introduce bias. Additionally, our survey questions were not psychometrically tested.

In summary, there is great variability in IM core clerkship models in the current era. Despite evidence of the value of a robust IM curriculum, the overall exposure to IM clinical training has declined. Future curriculum reform should consider measuring the effects of these changes on graduates. Advocacy for preserving IM clinical experiences will be critical to prevent future erosion of clinical IM in UME and to ensure that the highly valuable competencies taught and assessed uniquely by IM will not be neglected in developing generations of physicians.

Acknowledgments: The authors would like to thank the CDIM Survey and Scholarship Committee, CDIM Council members, and all CDIM members.

Corresponding Author: Amber T. Pincavage, MD; Department of Medicine, University of Chicago Pritzker School of Medicine, $5841 \mathrm{~S}$ Maryland Ave MC 3051, Chicago, IL 60637, USA (e-mail: apincava@medicine.bsd.uchicago.edu).

\section{Compliance with Ethical Standards:}

The survey protocol was granted full institutional review board exempt status from the University of North Carolina Office of Human Research Ethics.

Conflict of Interest: The authors declare that they do not have a conflict of interest.

Publisher's Note: Springer Nature remains neutral with regard to jurisdictional claims in published maps and institutional affiliations.

\section{REFERENCES}

1. The Complexities of Physician Supply and Demand Projections from 2016 to 2030. Final Report. March 2018. Prepared for Association of American Medical Colleges. Submitted by IHS Markit Ltd.

2. A Word from the President: Facing the Facts About the Physician Shortage. AAMC Reporter: December 2013. https://www.amc.org/ about/leadership/kirch-word-from-president/363844/word.html. Accessed January 29, 2019

3. Applicants and Matriculants (2018). AAMC data from 2008-2017. https://www.aamc.org/data/facts/applicantmatriculant/. Accessed January 29, 2019.

4. LCME: Functions and Structure of a Medical School. 2017. Standards for Accreditation of Medical Education Programs Leading to the MD Degree. http://lcme.org/publications/. Accessed January 29, 2019.

5. Cooke M, Irby D, Sullivan W, Ludmerer $\mathbf{K}$. American Medical Education 100 years after the Flexner Report. N Engl J Med. 2006; 355(13):13391344 
6. Brauer DG, Ferguson KJ. The integrated curriculum in medical education: AMEE Guide No. 96. Medical Teacher. 2015, 37:312-322.

7. Kevin Krane, MD. Curriculum Inventory in Context. https://www.aamc org/download/464758/data/ciic03-1jan2016 January 2016 Volume 3, Issue 1. P 1-3. Accessed January 29, 2019.

8. AAMC. The Core Entrustable Professional Activities (EPAs) for Entering Residency. 2014. https://www.aamc.org/initiatives/coreepas/publicationsandpresentations. Accessed January 29, 2019.

9. Bowen JL, Irby DM. Assessing quality and costs of education in the ambulatory setting: a review of the literature. Acad Med. 2002; 77(7):621680.

10. Levinsky NG. A survey of changes in the proportions of ambulatory training in internal medicine clerkships and residencies from 1986-87 to 1996-97. Acad Med.1998; 73(10):1114-1115.

11. Rosebraugh CJ, Szauter K, Ainsworth MA, Solomon DJ, Speer AJ, DiPette DJ. Creating and evaluating an independent ambulatory internal medicine clerkship. Am J Med Sci. 1998;315(1):30-4.

12. Shaheen A, Papp KK, Torre D. The internal medicine clerkship and ambulatory learning experiences: results of the 2010 clerkship directors in internal medicine survey. Teach Learn Med. 2013;25(3):225-230.

13. Hirsh DA, Ogur B, Thibault GE, Cox M. "Continuity" as an organizing principle for clinical education reform. N Engl J Med. 2007;356(8):858866.

14. Hauer KE, O'Brien B, Poncelet AN. Longitudinal, integrated clerkship education: better for learners and patients. Acad Med. 2009;84(7):821.

15. Norris TE, Schaad DC, DeWitt D, Ogur B, Hunt DD, Consortium of Longitudinal Integrated Clerkships. Longitudinal integrated clerkships for medical students: an innovation adopted by medical schools in Australia, Canada, South Africa, and the United States. Acad Med. 2009; 84(7):902-7.

16. Hirsh DA, Holmboe ES, ten Cate O. Time to trust: longitudinal integrated clerkships and entrustable professional activities. Acad Med. 2014;89(2):201-4

17. Hemmer PA, Ibrahim T, Durning SJ. The impact of increasing medical school class size on clinical clerkships: A national survey of internal medicine clerkship directors. Acad Med. 2008;83(5):432-437

18. Vu TR, Angus SV, Aronowitz PB, Harrell HE, Levine MA, Carbo A Whelton S, Ferris A, Appelbaum JS, McNeill DB, Ismail NJ, Elnicki DM. The internal medicine subinternship- now more important than ever: a joint CDIM-APDIM position paper. J Gen Intern Med. 2015; 30: 1369-7.

19. Sklar DS. Making the fourth year more meaningful. Acad Med. 2014; 89: 527-528.

20. Cosgrove EM, Ryan MJ, Wenrich MD. Empowering fourth-year medical students: the value of the senior year. Acad Med. 2014; 89(4): 533-535.

21. Stevens CD. Taking back year 4: a call to action. Acad Med. 2010; 85: 1663-1664.

22. Hemmer PA, Griffith C, Elnicki DM, Fagan M. The internal medicine clerkship in the clinical education of medical students. Am J Med. 2003; 115: 423-7
23. Aiyer MK, Vu TR, Ledford C, Fischer M, Durning SJ. The subinternship curriculum in internal medicine: a national survey of clerkship directors. Teach Learn Med. 2008;20(2):151-156.

24. StataCorp. 2015. Stata Statistical Software: Release 14. College Station, TX: StataCorp LP.

25. Liaison Committee on Medical Education (LCME). 2017. Medical School Directory. http://lcme.org/directory. Accessed January 29, 2019.

26. U.S. Census Bureau. Available at: www.census.gov/geo/maps-data/ reference.html Accessed January 29, 2019.

27. Barzansky B, Etzel SI. Educational Programs in US Medical Schools, 2002-2003. JAMA. 2003;290(9):1190-1196.

28. Fazio SB, Chheda S, Hingle S, Lo MC, Meade L, Blanchard M, Hoellein A, Brandenburg S, Denton GD. The Challenges of Teaching Ambulatory Internal Medicine: Faculty Recruitment, Retention, and Development: An AAIM/SGIM Position Paper. Am J Med. 2017;130(1):105-110.

29. Christner JG, Dallaghan GB, Briscoe G, Casey P, Fincher RM, Manfred LM, Margo KI, Muscarella P, Richardson JE, Safdieh J, Steiner BD. The Community Preceptor Crisis: Recruiting and Retaining CommunityBased Faculty to Teach Medical Students-A Shared Perspective From the Alliance for Clinical Education. Teach Learn Med. 2016;28(3):329-336.

30. Sidlow $\mathbf{R}$. The Structure and Content of the medical subinternship: a national survey. J Gen Intern Med. 2001; 16: 550-553.

31. Lyss-Lerman P, Teherani A, Aargard E, Loeser H, Cooke M, Harper GM. What training is needed in the fourth year of medical school? Views of resident program directors. Acad Med. 2009; 84: 823-829.

32. Pereira AG, Harrell HE, Weissman A, Smith CD, Dupras D, Kane GC. Important skills for internship and the fourth-year medical school courses to acquire them: a national survey of internal medicine residents. Acad Med. 2016; 91: 821-826.

33. Goren EN, Leizman DS, La Rochelle J, Kogan JR. Overnight Hospital Experiences for Medical Students: Results of the 2014 Clerkship Directors in Internal Medicine National Survey. J Gen Intern Med. 2015; 30(9): 1245-1250. Published online 2015 Jul 15. doi: https:// doi.org/10.1007/s11606-015-3405-4

34. ACGME 2011 duty hours standards. https://www.acgme.org/Portals/0/ PFAssets/ProgramResources/Common_Program_Requirements_ 07012011.pdf?ver=2015-11-06-120650-183 pp 31 and appendix E. Accessed January 29, 2019.

35. Oshimura JM, Sperring J, Bauer BD, Carroll AE, Rauch DA. Changes in inpatient staffing following implementation of new residency work hours. J Hosp Med. 2014; 9(10):640-5.

36. ACGME Summary of Changes to Common Program Requirements 2017. https://www.acgme.org/What-We-Do/Accreditation/Common-ProgramRequirements/Summary-of-Proposed-Changes-to-ACGME-CommonProgram-Requirements-Section-VI. Section VI.F. Accessed January 29, 2019.

37. Weinberger SE, Smith LG, Collier VU, Education Committee of the American College of PRedesigning training for internal medicine. Ann Intern Med. 2006; 144(12):927-932. 